\title{
Short- and long-lasting tinnitus relief induced by transcranial direct current stimulation
}

\author{
Pierre Garin • Chantal Gilain · Jean-Philippe Van Damme · Katalin de Fays • \\ Jacques Jamart $\cdot$ Michel Ossemann $\cdot$ Yves Vandermeeren
}

Received: 1 March 2011/Revised: 30 March 2011/Accepted: 31 March 2011 / Published online: 21 April 2011

(C) The Author(s) 2011. This article is published with open access at Springerlink.com

\begin{abstract}
A significant proportion of the population suffers from tinnitus, a bothersome auditory phantom perception that can severely alter the quality of life. Numerous experimental studies suggests that a maladaptive plasticity of the auditory and limbic cortical areas may underlie tinnitus. Accordingly, repetitive transcranial magnetic stimulation (rTMS) has been repeatedly used with success to reduce tinnitus intensity. The potential of transcranial direct current stimulation (tDCS), another promising method of noninvasive brain stimulation, to relieve tinnitus has not been explored systematically. In a double-blind, placebo-controlled and balanced order design, 20 patients suffering from chronic untreatable tinnitus were submitted to 20 minutes of $1 \mathrm{~mA}$ anodal, cathodal and sham tDCS
\end{abstract}

P. Garin · C. Gilain · J.-P. Van Damme

Centre Universitaire d'Audiophonologie,

Department of Otorhinolaryngology,

CHU Mont-Godinne, Université Catholique de Louvain (UCL),

Avenue Dr G. Therasse, 5530 Yvoir, Belgium

P. Garin

Department of Anatomy, Faculty of Medicine,

University of Namur, FUNDP, Namur, Belgium

K. de Fays · M. Ossemann · Y. Vandermeeren $(\bowtie)$

Department of Neurology, CHU Mont-Godinne,

Avenue Dr G. Therasse, 5530 Yvoir, Belgium

e-mail: yves.vandermeeren@uclouvain.be

K. de Fays · M. Ossemann · Y. Vandermeeren

Institute of Neuroscience (IoNS),

Université Catholique de Louvain (UCL),

Avenue Hippocrate, 54 Bte 54.10, 1200 Brussels, Belgium

J. Jamart

Scientific Support Unit, CHU Mont-Godinne,

5530 Yvoir, Belgium targeting the left temporoparietal area. The primary outcome measure was a change in tinnitus intensity or discomfort assessed with a Visual Analogic Scale (VAS) change-scale immediately after tDCS and 1 hour later. Compared to sham tDCS, anodal tDCS significantly reduced tinnitus intensity immediately after stimulation; whereas cathodal tDCS failed to do so. The variances of the tinnitus intensity and discomfort VAS change-scales increased dramatically after anodal and cathodal tDCS, whereas they remained virtually unchanged after sham tDCS. Moreover, several patients unexpectedly reported longer-lasting effects (at least several days) such as tinnitus improvement, worsening, or changes in tinnitus features, more frequently after real than sham tDCS. Anodal tDCS is a promising therapeutic tool for modulating tinnitus perception. Moreover, both anodal and cathodal tDCS seem able to alter tinnitus perception and could, thus, be used to trigger plastic changes.

Keywords Tinnitus - Transcranial direct current stimulation - Repetitive transcranial magnetic stimulation . Noninvasive brain stimulation - Brain plasticity

\section{Introduction}

Tinnitus is a bothersome auditory phantom perception (i.e., hearing a noise without an external source of sound) that can affect $10-15 \%$ of the general population [12]. Tinnitus can be a devastating pathology since a significant proportion of tinnitus sufferers develop sleep disturbances, depression, and other psychiatric comorbidities, and a small fraction commit suicide [8, 12]. Numerous drug regimens, behavioral therapies or noise-masking devices have been tried with an overall relatively disappointing 
lack of universal efficacy $[7,21]$. Since there is currently no satisfactory treatment for tinnitus, the condition of these patients may be especially distressing.

This lack of efficient therapies for tinnitus partly originates from an incomplete understanding of the pathophysiology of tinnitus, which is only starting to be unveiled. Whereas an initial event in the inner ear or in peripheral auditory pathways may initiate tinnitus, current hypotheses suggest that tinnitus could be maintained by the development of an abnormal plasticity triggered by deafferentation in a wide network of cortical areas and possibly subcortical structures $[6,23,32]$. Studies in animal models and patients have demonstrated both loss of hair cells in the inner hear and abnormal plastic alterations of tonotopic maps in the auditory cortex $[25,31,32]$, with the now classical "edge effect" which could be one of the mechanisms underlying tinnitus. A zone of the auditory cortex that used to represent a frequency band lost by deafferentation could be "invaded" by the adjacent frequency bands, leading to an enduring auditory phantom perception, i.e., to tinnitus [25, 32]. When comparing tinnitus patients with healthy volunteers, functional brain imaging studies using positron emission tomography (PET) or functional magnetic resonance imaging (fMRI) have consistently demonstrated abnormalities of the resting metabolism or activation in several brain structures, including the primary and secondary auditory cortices, limbic and emotional or attentional areas such as the dorsolateral prefrontal cortex, and subcortical structures [23-25]. Whereas the abnormal activity disclosed in the auditory-related areas (primary and secondary auditory cortices) could underlie the phantom auditory perception itself, the abnormal activity observed in the areas supporting cognitive, attentional, and limbic processes could be involved in the unpleasant and distressing aspect of tinnitus [31, 32]. Evoked auditory potentials or magnetoencephalography also point toward the persistence of an abnormal electric activity in a distributed network in tinnitus patients [34, 39].

The discovery of a disordered excitability in the auditory pathways and maladaptive plastic changes in auditory and limbic cortical areas led to the exciting hypothesis that it should be possible to treat tinnitus by modulating these abnormalities through brain stimulations. Indeed, several studies demonstrated that a single session of repetitive transcranial magnetic stimulation (rTMS) induces a transient relief of tinnitus [9, 29]. However, the therapeutic effect of rTMS waned as time elapsed. Therefore, aiming to extend this transient beneficial effect, several teams successfully applied repeated sessions of rTMS and induced a prolonged relief in tinnitus patients $[15,17,30$, 33]. Direct electric brain stimulation with epidural electrodes implanted over the auditory cortical areas has also been applied successfully in tinnitus patients; however, this approach requires invasive neurosurgical procedures [5].

Ten years ago, another method of noninvasive transcranial brain stimulation re-emerged after a long eclipse period: transcranial direct current stimulation (tDCS) [27]. Transcranial direct current stimulation has been extensively used to explore the neurophysiological mechanisms that govern human brain plasticity, as well as for modulating brain excitability. When applied over the primary motor cortex (M1), anodal (cathodal) tDCS increases (decreases) M1 excitability beyond the period of stimulation [27]. Transcranial direct current stimulation is considered as a promising therapeutic tool for several neurological and psychiatric pathologies which are driven by or result from abnormal brain excitability such as stroke, Parkinson's disease, chronic pain, and depression [10].

To date, only two studies explored the potential of tDCS to modulate tinnitus perception or discomfort: a pilot study targeting the left temporoparietal area (LTA) in 7 patients, with a washout period of a few minutes [9]; and a large study $(n=543)$ without a sham condition [37]. Since tDCS is easy to apply, is less expensive than rTMS, can be manipulated to design a high-quality sham condition necessary for therapeutic trials, and has-so far-not induced epileptic seizure, tDCS seems closer than rTMS in making the translation from bench to bedside, which is why we explored the therapeutic potential of tDCS in patients suffering from intractable tinnitus.

\section{Patients and method}

The research protocol was approved by the local ethical committee and conducted according to the recommendations of the Helsinki declaration. Tinnitus patients were recruited in the otorhinolaryngology outpatient consultation. Since we postulated that tinnitus is based on an abnormal plasticity triggered by auditory deafferentation, we included patients in whom a cochlear lesion could be objectified by hearing loss. Inclusion criteria were the following: (1) tinnitus that could not be cured by other means, (2) stable tinnitus for at least 2 months, (3) age 18-80 years, and (4) stable hypoacousia. Exclusion criteria were (1) Ménière's disease or fluctuating audition, (2) pure transduction hearing loss, (3) hyperacousia, (4) major cognitive impairment or psychiatric disorders, (5) severe comorbidity (e.g., heart failure, unstable diabetes), (6) contraindications to tDCS, including epilepsy, (7) chronic intake of alcohol or drugs that chronically affect brain functions (e.g., antidepressants, antiepileptics) stopped less than 1 month ago, and (8) pregnancy. 
The study assumed a double-blind, placebo-controlled, cross-over design. After a baseline evaluation with questionnaires (see below) at the otorhinolaryngology outpatient consultation, each patient underwent three sessions of tDCS (anodal, cathodal, and sham) in a balanced order, at 2 week intervals. Two weeks after the last tDCS session, the patients were evaluated again during a follow-up visit. A total of 21 tinnitus patients ( 5 females) signed informed consent and were enrolled after a structured interview as suggested by the Tinnitus Research Initiative (TRI) [20].

Transcranial direct current stimulation was delivered by an Eldith DC-Stimulator ${ }^{\circledR}$ (NeuroConn, Ilmenau, Germany) through electrodes embedded in sponges soaked with $\mathrm{NaCl} 0.9 \%$. The target cortical area was the left temporoparietal area (LTA), defined as being halfway between $\mathrm{C} 3$ and T5 measured with the 10-20 international EEG system [9, 29]. A $35 \mathrm{~cm}^{2}$ electrode was positioned over the LTA and a $50 \mathrm{~cm}^{2}$ electrode was positioned on the right scalp between T4 and F8. In order to guarantee the double-blind aspect of the experiment, an experimenter initially introduced a real or sham code for each session. The order of the codes was balanced across patients and sessions. A second (blinded) experimenter collected the behavioral data. During real (anodal/cathodal) stimulation, the Eldith DC-Stimulator delivered 20 minutes of DC stimulation at $1 \mathrm{~mA}(1 \mathrm{~mA}$ plateau, fade in/out $8 \mathrm{~s})$. For sham tDCS, the polarity of the LTA electrode was anodal in $50 \%$ of the patients; after a short up-ramp, brief current pulses $(110 \mu \mathrm{A}$ over $15 \mathrm{~ms}$, peak current $3 \mathrm{~ms})$ were delivered every $550 \mathrm{~ms}$, eliciting a tingling sensation similar to that felt during real stimulation.

The primary outcome measure was a change in tinnitus intensity or discomfort assessed with a Visual Analogic Scale (VAS) change-scale immediately after tDCS and 1 hour later. The VAS change-scale ranged from "full relief" $(+4)$ to "very strong deterioration" $(-4)$, with 0 being "unchanged". Secondary outcome measures included potential long-term effects over the 2 weeks following each tDCS session on a French translation of the Tinnitus Questionnaire (TQ) [11, 22] and the Beck Depression Inventory (BDI) [2], as well as free reports from patients. The TQ, BDI, and free reports were collected at inclusion and follow-up consultations, and before each tDCS session. The tinnitus patients were dichotomized into a "low, compensated" (TQ < 46) or "high, decompensated" (TQ $\geq 47$ ) distress category, according to the burden of psychological and depressive features [38].

For statistical analysis, the VAS change-scales for tinnitus intensity and discomfort immediately and 1 hour after tDCS sessions, the TQ and BDI scores, were compared between anodal, cathodal and sham tDCS by Friedman test, followed in case of a statistically significant heterogeneity by $2 \times 2$ Wilcoxon signed rank tests. The Pitman-Morgan test using the Spearman correlation coefficient was used for comparing the variances of sham versus anodal, sham versus cathodal and anodal versus cathodal. Behavioral effects spontaneously reported were compared between anodal, cathodal, and sham tDCS by $2 \times 2$ binomial tests. All statistical tests are two-tailed and were performed by SPSS 15.0 statistical software (SPSS Inc., Chicago, IL, USA).

\section{Results}

One depressive female patient was excluded because she needed to start antidepressant therapy between the first and second tDCS sessions. The characteristics of the 20 patients who completed the study are listed in Table 1. The age of the patients was $50.9 \pm 12.9$ years; they suffered from tinnitus for at least 8 months. After dichotomization into distress category, the TQ scores were $36.4 \pm 10.2$ in compensated (TQ $<46, n=13$ ) and $56.6 \pm 9.8$ in decompensated (TQ $\geq 47, n=7$ ) tinnitus patients.

Transcranial direct current stimulation was well tolerated. One of the patients (\#19) reported a transient instability after sham tDCS which resolved within 1 hour. Some patients mentioned transient or longer-lasting tinnitus worsening, mostly under cathodal tDCS (see below).

A global analysis demonstrated a significant effect ( $p=0.013$ ) for tinnitus intensity immediately after tDCS. When compared to sham tDCS, the VAS change-scale for tinnitus intensity significantly decreased immediately after anodal tDCS $(p=0.020)$. There was no statistically significant difference between sham and cathodal $(p=0.414)$ nor between anodal and cathodal tDCS $(p=0.132)$. One hour after tDCS, the VAS change-scale for intensity was not different anymore between anodal, cathodal, and sham tDCS $(p=0.543)$. Whereas, the temporal evolution of the VAS change-scales for discomfort were similar to those for tinnitus intensity (Fig. 1), there was no significant difference immediately after anodal, cathodal, or sham tDCS $(p=0.108)$, nor 1 hour later $(p=0.761)$.

While 7 patients were positive responders to anodal tDCS, 4 other patients reported a slight tinnitus worsening after anodal tDCS. Six patients were positive responders to cathodal tDCS; 5 patients were also positive responders to anodal tDCS. Five others patients reported slight tinnitus worsening after cathodal tDCS, 1 patient responded positively to anodal tDCS, and 2 patients experienced worsening after anodal tDCS.

As shown in Fig. 1, the temporal evolution of the variances of the tinnitus intensity and discomfort VAS changescales were strikingly different under real (anodal and cathodal) and sham tDCS. Indeed, the variance remained 
Table 1 Demographic data of patients and their tinnitus characteristics

\begin{tabular}{|c|c|c|c|c|c|c|c|c|c|c|}
\hline & $\begin{array}{l}\text { Age } \\
\text { (years) }\end{array}$ & Sex & $\begin{array}{l}\text { Duration } \\
\text { (years) }\end{array}$ & Origin & Laterality $^{\mathrm{a}}$ & $\begin{array}{l}\text { Class of hearing } \\
\text { loss right/left }\end{array}$ & $\begin{array}{l}\text { Auditory curve } \\
\text { right/left }^{\mathrm{c}}\end{array}$ & $\begin{array}{l}\text { Mean } \\
\text { BDI }\end{array}$ & $\begin{array}{l}\text { Mean } \\
\text { TQ }\end{array}$ & Compens \\
\hline 1 & 48 & M & $1-2$ & Early presbyacusis & $\mathrm{R}=\mathrm{L}$ & $\mathrm{L} / \mathrm{L}$ & $\mathrm{d} / \mathrm{d}$ & 5.3 & 55.5 & $\mathrm{D}$ \\
\hline 2 & 23 & M & $5-10$ & Unknown & $\mathrm{L}$ & $\mathrm{P} / \mathrm{L}$ & $-/ a$ & 1.0 & 39.0 & $\mathrm{C}$ \\
\hline 3 & 29 & $\mathrm{~F}$ & $>10$ & $\begin{array}{l}\text { Otosclerosis (having had } \\
\text { surgical treatment) }\end{array}$ & Head & $\mathrm{L} / \mathrm{M}$ & $-/ \mathrm{d}$ & 4.0 & 44.0 & $\mathrm{C}$ \\
\hline 4 & 45 & M & $1-2$ & Sudden hearing loss & $\mathrm{L}$ & $\mathrm{N} / \mathrm{N}$ & $\mathrm{s} / \mathrm{s}$ & 1.0 & 18.5 & $\mathrm{C}$ \\
\hline 5 & 59 & M & $<1$ & Presbyacusis & $\mathrm{R}=\mathrm{L}$ & $\mathrm{N} / \mathrm{N}$ & $\mathrm{s} / \mathrm{s}$ & 1.5 & 48.0 & $\mathrm{D}$ \\
\hline 6 & 54 & M & $>10$ & Acoustic trauma & $\mathrm{L}$ & $\mathrm{L} / \mathrm{L}$ & $\mathrm{d} / \mathrm{d}$ & 14.0 & 39.0 & $\mathrm{C}$ \\
\hline 7 & 65 & M & $1-2$ & Presbyacusis & $\mathrm{R}$ & $\mathrm{L} / \mathrm{L}$ & $\mathrm{d} / \mathrm{d}$ & 0.5 & 42.0 & $\mathrm{C}$ \\
\hline 8 & 67 & M & $2-5$ & Presbyacusis & $\mathrm{L}$ & $\mathrm{L} / \mathrm{L}$ & $\mathrm{d} / \mathrm{d}$ & 1.0 & 15.5 & $\mathrm{C}$ \\
\hline 9 & 55 & M & $5-10$ & Presbyacusis + acoustic trauma & $\mathrm{R}=\mathrm{L}$ & $\mathrm{L} / \mathrm{L}$ & $\mathrm{d} / \mathrm{d}$ & 4.5 & 56.0 & $\mathrm{D}$ \\
\hline 10 & 44 & M & $2-5$ & Unknown & $\mathrm{L}$ & $\mathrm{N} / \mathrm{N}$ & $\mathrm{s} / \mathrm{s}$ & 0.5 & 24.5 & $\mathrm{C}$ \\
\hline 11 & 49 & M & $>10$ & Presbyacusis + whiplash & $\mathrm{R}=\mathrm{L}$ & $\mathrm{L} / \mathrm{L}$ & $d / d$ & 4.3 & 42.0 & $\mathrm{C}$ \\
\hline 12 & 75 & M & $>10$ & Presbyacusis & $\mathrm{L}$ & $\mathrm{M} / \mathrm{M}$ & $\wedge / \mathrm{d}$ & 7.0 & 45.0 & $\mathrm{C}$ \\
\hline 13 & 56 & M & $>10$ & Acoustic trauma & $\mathrm{R}=\mathrm{L}$ & $\mathrm{N} / \mathrm{N}$ & $\mathrm{a} /-$ & 5.5 & 44.5 & $\mathrm{C}$ \\
\hline 14 & 61 & M & $5-10$ & Presbyacusis & Head & $\mathrm{M} / \mathrm{L}$ & $\mathrm{d} / \mathrm{d}$ & 27.0 & 78.0 & $\mathrm{D}$ \\
\hline 15 & 51 & $\mathrm{~F}$ & $5-10$ & Presbyacusis & $\mathrm{R}>\mathrm{L}$ & $\mathrm{L} / \mathrm{L}$ & $\wedge / \wedge$ & 8.0 & 55.0 & $\mathrm{D}$ \\
\hline 16 & 48 & M & $1-2$ & Acoustic trauma + whiplash & $\mathrm{R}<\mathrm{L}$ & $\mathrm{L} / \mathrm{N}$ & $\mathrm{d} / \mathrm{d}$ & 2.5 & 43.5 & $\mathrm{C}$ \\
\hline 17 & 60 & M & $<1$ & Presbyacusis + acoustic trauma & $\mathrm{R}>\mathrm{L}$ & $\mathrm{L} / \mathrm{N}$ & $-/ \mathrm{d}$ & 8.0 & 51.0 & $\mathrm{D}$ \\
\hline 18 & 35 & $\mathrm{~F}$ & $>10$ & Unknown & Head & $\mathrm{N} / \mathrm{N}$ & $\mathrm{d} /-$ & 1.5 & 40.0 & $\mathrm{C}$ \\
\hline 19 & 57 & M & $1-2$ & Presbyacusis + whiplash & $\mathrm{R}=\mathrm{L}$ & $\mathrm{N} / \mathrm{N}$ & $d / d$ & 3.0 & 53.0 & $\mathrm{D}$ \\
\hline 20 & 37 & $\mathrm{~F}$ & $>10$ & Whiplash & $\mathrm{R}>\mathrm{L}$ & $\mathrm{N} / \mathrm{N}$ & $-1-$ & 2.8 & 35.5 & $\mathrm{C}$ \\
\hline
\end{tabular}

Mean BDI (Beck Depression Inventory)/mean TQ (Tinnitus Questionnaire) scores at baseline (mean of the scores at the NET visit and inclusion); compens compensated tinnitus, $C$ compensated tinnitus (TQ $<46), D$ decompensated tinntitus (TQ $>47$ )

${ }^{a}$ Laterality: $R$ right tinnitus, $L$ left tinnitus, $R=L$ bilateral tinnitus, $R>L$ bilateral tinnitus with right predominance, $R<L$ bilateral tinnitus with left predominance

${ }^{\mathrm{b}}$ Class of hearing loss (mean of hearing loss at 1,000, 2,000 and 4,000 Hz); $N$ normal hearing threshold $(<20 \mathrm{~dB})$ on average but with a hearing loss at least over a frequency range, $L$ mild hearing loss (20-40 dB), $M$ moderate hearing loss (41-70 dB), $S$ severe hearing loss (70-90 db), $P$ profound hearing loss $(>90 \mathrm{db})$

c Auditory curves; $d$ descending, $a$ ascending, - horizontal, $\wedge$ inverted $\mathrm{v}$-shape, $s$ scotoma on 4 or $6 \mathrm{kHz}$

Fig. 1 Plot of the (mean $\pm \mathrm{SD}$ ) group effects of sham, anodal and cathodal tDCS sessions immediately and 1 hour after tDCS on tinnitus a intensity and $\mathbf{b}$ discomfort (VAS change scale).

$* * p=0.020, * p>0.132$
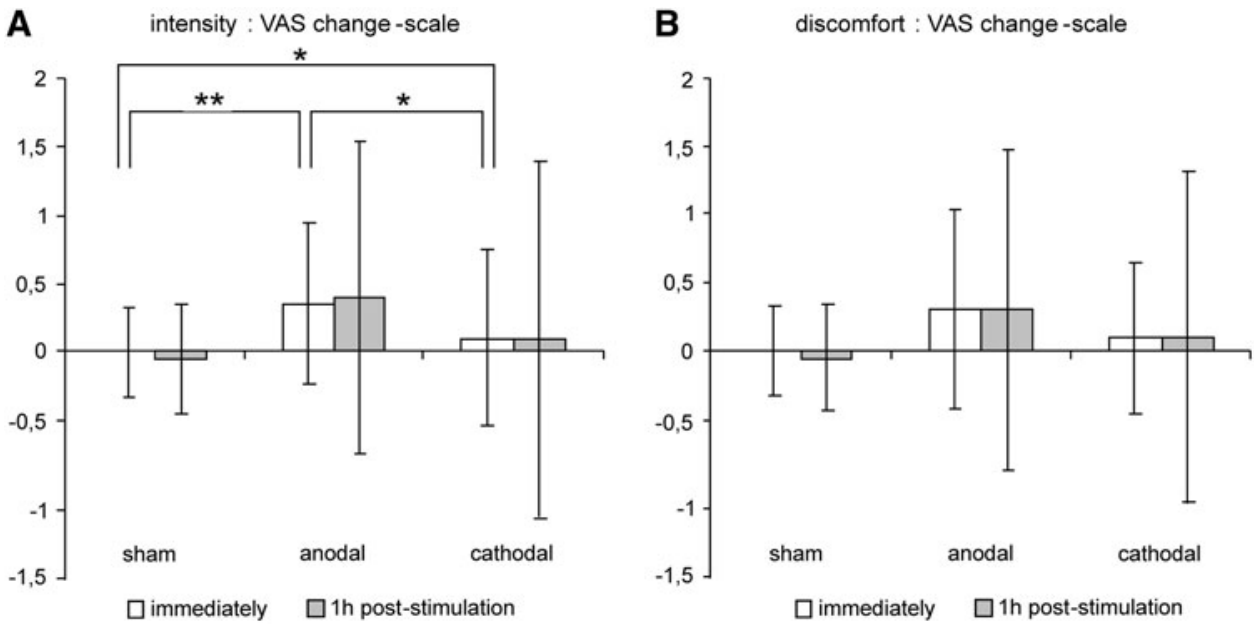

virtually unchanged after sham tDCS but increased dramatically after anodal and cathodal tDCS. When compared to sham tDCS, the variances of the intensity VAS change- scales increased immediately after anodal and cathodal tDCS ( $p=0.011$ and $p<0.001$, respectively) and kept increasing 1 hour later $(p=0.004$ and $p=0.001)$. There 
Table 2 Long-lasting effects of tDCS sessions reported spontaneously by tinnitus patients

\begin{tabular}{|c|c|c|c|}
\hline & Sham & Anodal & Cathodal \\
\hline 3 & & Improvement (intensity and discomfort) during 2 days & $\begin{array}{l}\text { Tone different, higher pitch, balance } \\
\text { left/right }\end{array}$ \\
\hline 5 & & Worsening (intensity and discomfort) during 10 days & $\begin{array}{l}\text { During a few days: decreased intensity on the } \\
\text { right side, possible increase on the left }\end{array}$ \\
\hline 7 & $\begin{array}{l}\text { Slight increase of intensity and } \\
\text { higher pitch with less variation, } \\
\text { during a few days }\end{array}$ & $\begin{array}{l}\text { Worsening (discomfort) } \geq 15 \text { days; very high pitch, lack } \\
\text { of the tone variations }\end{array}$ & \\
\hline 9 & & Worsening (intensity and discomfort) during 7 days & $\begin{array}{l}\text { Worsening (intensity and discomfort) } \\
\geq 15 \text { days, lack of the tone variations }\end{array}$ \\
\hline 10 & $\begin{array}{l}\text { Increase of intensity } \\
\text { during } 7 \text { days }\end{array}$ & $\begin{array}{l}\text { Improvement (intensity and discomfort) during } \\
\geq 15 \text { days }\end{array}$ & $\begin{array}{l}\text { Improvement (intensity and discomfort) } \\
\geq 15 \text { days; lower tone less uncomfortable }\end{array}$ \\
\hline 13 & & $\begin{array}{l}\text { Clear "well-being" during the afternoons during } \\
\geq 15 \text { days }\end{array}$ & \\
\hline 15 & & $\begin{array}{l}\text { During a few days: decrease of intensity on the left, } \\
\text { lower tone less uncomfortable }\end{array}$ & \\
\hline 16 & & $\begin{array}{l}\text { Complete but transient resolution of tinnitus a few hours } \\
\text { later, alternation of resolution/relapses, better mood }\end{array}$ & $\begin{array}{l}\text { Worsening (intensity and discomfort) during } \\
\geq 15 \text { days }\end{array}$ \\
\hline 17 & & $\begin{array}{l}\text { Modulation of intensity, slight pain, progressive return } \\
\text { to previous status }\end{array}$ & \\
\hline 19 & $\begin{array}{l}\text { Transient instability after sham } \\
\text { tDCS resolved in } 1 \text { hour; } \\
\text { increase of intensity }>1 \text { hour }\end{array}$ & & \\
\hline
\end{tabular}

Long-lasting positive, negative or other effects (change in pitch, tone, side balance, qualities of tinnitus) spontaneously reported by the patients after sham, anodal and cathodal tDCS. Patients $1,2,4,6,8,11,12,14,18$, and 20 did not report any lasting effects

was no statistically significant difference in the variances between anodal and cathodal tDCS $(p>0.283)$. For the discomfort VAS change-scale, the variances were not significantly different immediately after tDCS $(p>0.108)$. However, 1 hour later, the variance significantly increased after anodal $(p=0.004)$ and cathodal $(p=0.002)$ tDCS when compared to sham tDCS; again, there was no statistically significant difference between anodal and cathodal tDCS $(p=0.9)$.

Unexpectedly, several patients spontaneously reported either lasting changes of tinnitus characteristics (loudness, pitch, fluctuations, etc.) or improvement/worsening that persisted several days (see Table 2). After anodal tDCS, 6 patients reported prolonged positive effects, and 3 patients mentioned lasting negative effects. After cathodal tDCS, 2 patients reported a lasting negative effect and 3 patients a positive effect. After sham tDCS, 3 patients reported a protracted negative effect. Overall, patients reported long-lasting benefits more frequently after anodal than sham tDCS $(p=0.031)$, whereas there was no significant difference between sham and cathodal tDCS $(p=0.25)$, nor between anodal and cathodal tDCS $(p=0.375)$.

When comparing the TQ and BDI scores evaluating the 2 week epochs following each tDCS session, there was no statistically significant difference between sham, anodal, and cathodal tDCS (all $p>0.229$ ). However, as shown in
Fig. 2, the mean TQ score decreased steadily and significantly $(p=0.008)$ from baseline $(43.5 \pm 13.9)$ to followup $(37.3 \pm 16.1)$. The level of depression and anxiety (BDI scores, Fig. 2) remained unchanged over time $(p=0.299)$.

\section{Discussion}

The current study shows a net beneficial short-term effect of anodal tDCS applied over the left LTA on tinnitus intensity in 7 out of 20 patients ("positive responder" rate $35 \%$ ) and extends previous reports by suggesting unexpected longer-lasting effects of a single tDCS session. There was no difference in the TQ and BDI scores but the study was neither designed nor powered for that purpose. The decrease of TQ scores over time may reflect a socalled "Hawthorne effect" [3], i.e., a nonspecific improvement due to the patient's involvement into the study and the care devoted to follow their level of wellbeing.

Two key differences with previous tDCS studies $[9,37]$ were the experimental design and the electrode montage. First, the current experiment assumed a double-blind, placebo-controlled, and cross-over design with a long washout period ( 2 weeks). This temporal aspect is especially important since the recent history of cortical excitability can shape (and even reverse) the direction of excitability 
Fig. 2 Temporal evolution of the Tinnitus Questionnaire (TQ) scores and Beck Depression Inventory (BDI) scores from baseline to follow-up (mean $\pm \mathrm{SD}$ )
A

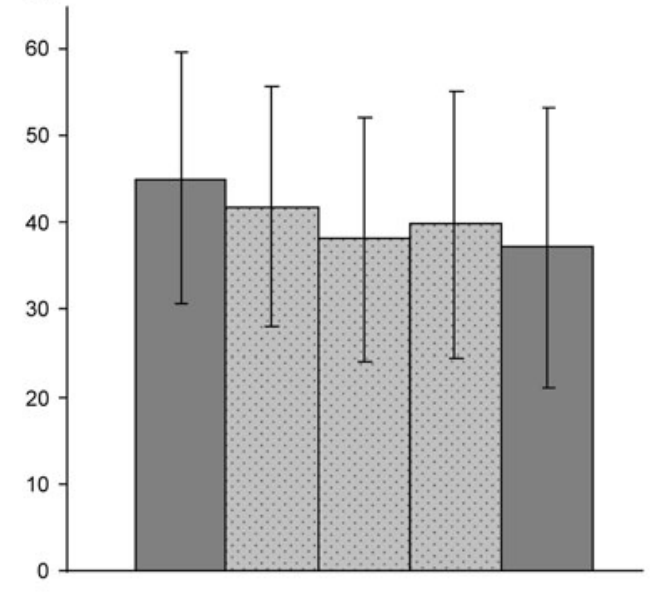

B

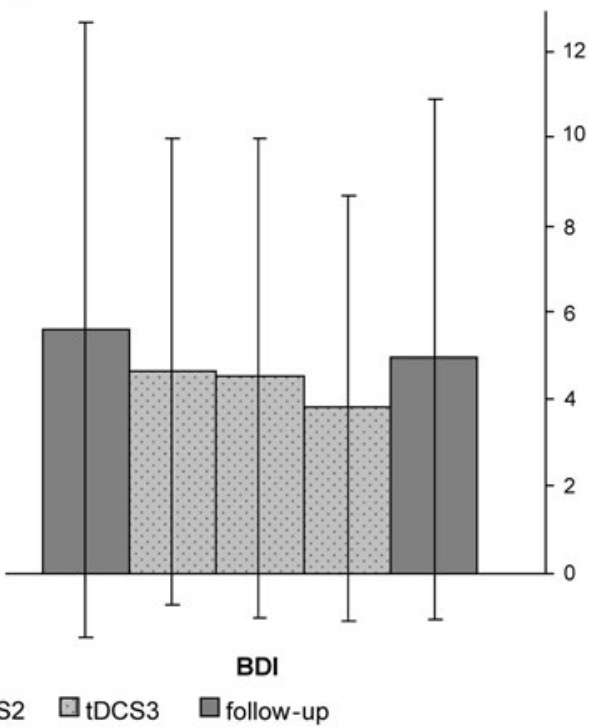

changes induced by tDCS or rTMS, this phenomenon is known as homeostatic plasticity or metaplasticity [40]. The second key difference with previous studies, in which the two electrodes were of the same size, is that we positioned a medium $\left(35 \mathrm{~cm}^{2}\right)$ electrode over the left LTA and a larger $\left(50 \mathrm{~cm}^{2}\right)$ electrode over the right laterofrontal scalp. Recent tDCS experiments have demonstrated that the excitability of a cortical area under a large electrode remains unchanged, ensuring that this large electrode could be considered as a real "neutral" reference electrode [26]. Using a smaller electrode targeting the cortical area of interest and a large reference electrode helps to disambiguate the interpretation of previous tDCS studies in which a combined effect from both "active" electrodes with opposite polarities could not be ruled out. Another way to avoid this ambiguity is to use an extracephalic reference electrode, which seems to be a safe alternative [35]. In the current experiment, the effects observed on tinnitus intensity may be chiefly driven by the DC stimulation of the left LTA; however, a significant contribution of the right frontal cognitive and limbic areas involved the emotional aspects of tinnitus [31] can not be ruled out.

Both our responder rate $35 \%$ of the patients were "positive responders" to anodal tDCS) and effect size were comparable to those reported in previous rTMS [19, 28] and tDCS studies $[9,37]$. Whereas relatively low, these responder rate and effect size still compare favorably with the lower rate of success of other therapeutic approaches. Moreover, it should be acknowledged that the therapeutic trials designed to modulate tinnitus with noninvasive transcranial brain stimulations are still in their infancy and that the optimal parameters (target area, intensity, duration, etc.) remain to be settled before launching large randomized control trials. Despite the current lack of such trial, some patient's characteristics seem to predict a positive response to rTMS, such as tinnitus duration and normal hearing [18], whereas age, gender, and tinnitus duration, type and laterality are not predictive for tDCS [37].

Whereas a significant beneficial effect was observed exclusively for anodal tDCS, it is, however, striking to compare the stability of the variance under sham tDCS with the dynamical evolution of the variances after both anodal and cathodal tDCS (Fig. 1). Two hypotheses could explain this observation. The first one is that patients became aware of the real/sham nature of the stimulations. In order to avoid drawing their attention on this aspect, we kept talking with them during stimulation. We did not ask specifically what their guess was; therefore, this possibility cannot be formally excluded. However, this is unlikely for several reasons. First, the stimulation parameters used are considered as "classical" in tDCS experiments and have been proven safe for patient's blinding, although a significant proportion of healthy volunteers may formulate a correct guess at $1 \mathrm{~mA} \mathrm{[1].} \mathrm{Second,} \mathrm{during} \mathrm{sham} \mathrm{tDCS,} \mathrm{the}$ Eldith DC-Stimulator ${ }^{\circledR}$ delivered brief and ineffective current pulses inducing a similar itching sensation that improves the blinding procedure. Third, the order of the stimulations was balanced across patients and sessions, which decreases the likelihood of unblinding all the patients. Fourth, the behavioral data were collected in a double-blind fashion. Finally, after sham tDCS, 4 of 20 patients reported short-lasting effects and 3 longer-lasting effects, suggesting they did not guess the sham nature of that stimulation. Thus, it is unlikely that the differential temporal evolution of the variance between sham and real tDCS could be explained by an unblinding of the patients. 
The second hypothesis to explain this increasing variance over time solely after real but not sham tDCS is that both anodal and cathodal tDCS interacted with the left LTA activity and triggered changes in tinnitus perception/ discomfort which built up over at least 1 hour, but with divergent effects (favorable or unfavorable) in different patients. At the group level, in addition to an increase of variance over time suggesting an ongoing dynamical phenomenon, anodal tDCS resulted in a net favorable effect, whereas cathodal tDCS did not. Besides the "canonical" effects on M1 excitability in humans [27], anodal and cathodal tDCS may both have interfered with an ongoing state of abnormal excitability such as observed in tinnitus patients $[31,32]$. This may be in line with the recent observation of large-scale network abnormalities as revealed by functional connectivity in tinnitus patients [36]. Given the fact that several patients also reported additional changes in their tinnitus features (pitch, loudness, laterality) after real tDCS, one could hypothesize that anodal and cathodal tDCS perturbed a maladaptive equilibrium state and opened the door for plastic changes to develop, some patients taking advantage and experiencing an improvement, while others reported a worsening or useless changes in tinnitus features. The reason why tDCS triggers beneficial changes in some patients and detrimental in others remains an open question; reminiscent of the issue why some patients respond to rTMS or tDCS and others do not. This could be related to the clinical history and tinnitus features [18, 37], to the particular excitability of network reorganization (more or less "limbic" or "auditory") [31, 32], the recent history of the LTA or connected areas excitability changes (homeostatic plasticity) [40], or to their genetic background such as the polymorphism of the brain-derived neurotrophic factor [4].

If real tDCS triggered spontaneous plastic changes by disrupting an ongoing state of abnormal excitability, this could also potentially explain the unexpected long-term effects on tinnitus spontaneously reported by our patients. Recent studies with repeated rTMS sessions have demonstrated a remarkably high success rate with long-term benefits [16]. It is worth noting that long-lasting beneficial effects on tinnitus have been induced by repeated sessions of either low-frequency ("inhibitory") rTMS or high-frequency ("excitatory") rTMS in a study with a parallel group design [13-15], suggesting that both low- and highfrequency rTMS could trigger plastic changes in tinnitus patients, just as we observed with a single session of anodal or cathodal tDCS.

It has been suggested that rTMS might exert a multimodal effect on tinnitus: directly on brain excitability and indirectly through acoustic stimuli and peripheral nerves stimulation [19]; however, the involvement of peripheral nerve stimulation is debated [33]. Transcranial direct current stimulation is less likely to benefit from such a potential multimodal effect since it does not deliver acoustic stimulation and since the itching sensation driven by DC stimulation of the scalp nerves rapidly fades. On the one hand, this may lessen the therapeutic potential of tDCS compared to rTMS. On the other hand, the conclusions from tDCS studies may be less contaminated by poorer sham conditions or multimodal effects.

\section{Conclusion}

In summary, both anodal and cathodal tDCS applied over the left LTA modulated the perceived tinnitus intensity when compared to sham tDCS; but solely anodal tDCS induced a significant tinnitus relief, whereas cathodal tDCS did not, despite the aforementioned dynamical modulation. These results demonstrate that tDCS can noninvasively modulate tinnitus perception. At first glance, these mixed results with tDCS may not seem encouraging from an individual patient's perspective since some patients do not respond to tDCS and others may experience (transient) tinnitus worsening. However, these dynamic changes after anodal and cathodal tDCS when compared to sham stimulation may also be interpreted as a potential therapeutic opportunity to seize. Indeed, if tDCS applied in tinnitus patients triggers excitability changes, this may open a window for brain plasticity that could potentially be tuned toward improvement; one of the goals of future studies should be to optimize tDCS parameters for relieving tinnitus.

Acknowledgments We would like to thank the patients who consented to take part to the experiment. We also would like to thank Professor Patrice Laloux for his unfailing support. Christian Deneffe's assistance with the figures is gratefully appreciated. The work of YV and the acquisition of the tDCS device were supported by the following grants: Fonds de la Recherche Scientifique Médicale FRSM 3.4.525.08.F 2008 and 2010; Fondation van Goethem-Brichant 2007 grant; Université Catholique de Louvain (UCL) Fonds Spécial de Recherche FSR 2008 and 2010.

Conflict of interest None.

Open Access This article is distributed under the terms of the Creative Commons Attribution Noncommercial License which permits any noncommercial use, distribution, and reproduction in any medium, provided the original author(s) and source are credited.

\section{References}

1. Ambrus GG, Paulus W, Antal A (2010) Cutaneous perception thresholds of electrical stimulation methods: comparison of tDCS and tRNS. Clin Neurophysiol 121(11):1908-1914

2. Beck AT, Ward CH, Mendelson M, Mock J, Erbaugh J (1961) An inventory for measuring depression. Arch Gen Psychiatry 4:561-571 
3. Campbell JP, Maxey VA, Watson WA (1995) Hawthorne effect: implications for prehospital research. Ann Emerg Med 26:590-594

4. Cheeran B, Talelli P, Mori F, Koch G, Suppa A, Edwards M, Houlden H, Bhatia K, Greenwood R, Rothwell JC (2008) A common polymorphism in the brain-derived neurotrophic factor gene (BDNF) modulates human cortical plasticity and the response to rTMS. J Physiol 586:5717-5725

5. De Ridder D, De Mulder G, Verstraeten E, Van der Kelen K, Sunaert S, Smits M, Kovacs S, Verlooy J, Van de Heyning P, Moller AR (2006) Primary and secondary auditory cortex stimulation for intractable tinnitus. ORL J Otorhinolaryngol Relat Spec 68:48-54 (discussion 54-55)

6. Eggermont JJ, Roberts LE (2004) The neuroscience of tinnitus. Trends Neurosci 27:676-682

7. Elgoyhen AB, Langguth B (2010) Pharmacological approaches to the treatment of tinnitus. Drug Discov Today 15:300-305

8. Frankenburg FR, Hegarty JD (1994) Tinnitus, psychosis, and suicide. Arch Intern Med 154:2371-2375

9. Fregni F, Marcondes R, Boggio PS, Marcolin MA, Rigonatti SP, Sanchez TG, Nitsche MA, Pascual-Leone A (2006) Transient tinnitus suppression induced by repetitive transcranial magnetic stimulation and transcranial direct current stimulation. Eur $\mathbf{J}$ Neurol 13:996-1001

10. Fregni F, Pascual-Leone A (2007) Technology insight: noninvasive brain stimulation in neurology-perspectives on the therapeutic potential of rTMS and tDCS. Nat Clin Pract Neurol 3:383-393

11. Goebel G, Hiller W (1994) The tinnitus questionnaire. A standard instrument for grading the degree of tinnitus. Results of a multicenter study with the tinnitus questionnaire. HNO 42:166-172

12. Henry JA, Dennis KC, Schechter MA (2005) General review of tinnitus: prevalence, mechanisms, effects, and management. J Speech Lang Hear Res 48:1204-1235

13. Khedr EM, Abo-Elfetoh N, Rothwell JC, El-Atar A, Sayed E, Khalifa H (2010) Contralateral versus ipsilateral rTMS of temporoparietal cortex for the treatment of chronic unilateral tinnitus: comparative study. Eur J Neurol 17:976-983

14. Khedr EM, Rothwell JC, Ahmed MA, Awad EM, Galal O (2008) Cortical excitability and transcallosal inhibition in chronic tinnitus: transcranial magnetic study. Neurophysiol Clin 38:243-248

15. Khedr EM, Rothwell JC, Ahmed MA, El-Atar A (2008) Effect of daily repetitive transcranial magnetic stimulation for treatment of tinnitus: comparison of different stimulus frequencies. J Neurol Neurosurg Psychiatry 79:212-215

16. Khedr EM, Rothwell JC, El-Atar A (2009) One-year follow up of patients with chronic tinnitus treated with left temporoparietal rTMS. Eur J Neurol 16:404-408

17. Kleinjung T, Eichhammer P, Langguth B, Jacob P, Marienhagen J, Hajak G, Wolf SR, Strutz J (2005) Long-term effects of repetitive transcranial magnetic stimulation (rTMS) in patients with chronic tinnitus. Otolaryngol Head Neck Surg 132:566-569

18. Kleinjung T, Steffens T, Sand P, Murthum T, Hajak G, Strutz J, Langguth B, Eichhammer P (2007) Which tinnitus patients benefit from transcranial magnetic stimulation? Otolaryngol Head Neck Surg 137:589-595

19. Langguth B, de Ridder D, Dornhoffer JL, Eichhammer P, Folmer RL, Frank E, Fregni F, Gerloff C, Khedr E, Kleinjung T, Landgrebe M, Lee S, Lefaucheur JP, Londero A, Marcondes R, Moller AR, Pascual-Leone A, Plewnia C, Rossi S, Sanchez T, Sand P, Schlee W, Pysch D, Steffens T, van de Heyning P, Hajak G (2008) Controversy: does repetitive transcranial magnetic stimulation/transcranial direct current stimulation show efficacy in treating tinnitus patients? Brain Stimul 1:192-205

20. Langguth B, Goodey R, Azevedo A, Bjorne A, Cacace A, Crocetti A, Del Bo L, De Ridder D, Diges I, Elbert T, Flor H, Herraiz C, Ganz Sanchez T, Eichhammer P, Figueiredo R, Hajak
G, Kleinjung T, Landgrebe M, Londero A, Lainez MJ, Mazzoli M, Meikle MB, Melcher J, Rauschecker JP, Sand PG, Struve M, Van de Heyning P, Van Dijk P, Vergara R (2007) Consensus for tinnitus patient assessment and treatment outcome measurement: Tinnitus Research Initiative meeting, Regensburg, July 2006. Prog Brain Res 166:525-536

21. Martinez Devesa P, Waddell A, Perera R, Theodoulou M (2007) Cognitive behavioural therapy for tinnitus. Cochrane Database Syst Rev:CD005233

22. Meeus O, Blaivie C, Van de Heyning P (2007) Validation of the Dutch and the French version of the Tinnitus Questionnaire. B-ENT 3(Suppl 7):11-17

23. Melcher JR, Sigalovsky IS, Guinan JJ Jr, Levine RA (2000) Lateralized tinnitus studied with functional magnetic resonance imaging: abnormal inferior colliculus activation. J Neurophysiol 83:1058-1072

24. Muhlau M, Rauschecker JP, Oestreicher E, Gaser C, Rottinger M, Wohlschlager AM, Simon F, Etgen T, Conrad B, Sander D (2006) Structural brain changes in tinnitus. Cereb Cortex 16:1283-1288

25. Muhlnickel W, Elbert T, Taub E, Flor H (1998) Reorganization of auditory cortex in tinnitus. Proc Natl Acad Sci USA 95:10340-10343

26. Nitsche MA, Doemkes S, Karakose T, Antal A, Liebetanz D, Lang N, Tergau F, Paulus W (2007) Shaping the effects of transcranial direct current stimulation of the human motor cortex. J Neurophysiol 97:3109-3117

27. Nitsche MA, Paulus W (2000) Excitability changes induced in the human motor cortex by weak transcranial direct current stimulation. J Physiol 527(Pt 3):633-639

28. Plewnia C (2010) Brain Stimulation: new vistas for the exploration and treatment of tinnitus. CNS Neurosci Ther (in press)

29. Plewnia C, Bartels M, Gerloff C (2003) Transient suppression of tinnitus by transcranial magnetic stimulation. Ann Neurol 53:263-266

30. Plewnia C, Reimold M, Najib A, Reischl G, Plontke SK, Gerloff C (2007) Moderate therapeutic efficacy of positron emission tomography-navigated repetitive transcranial magnetic stimulation for chronic tinnitus: a randomised, controlled pilot study. J Neurol Neurosurg Psychiatry 78:152-156

31. Rauschecker JP, Leaver AM, Muhlau M (2010) Tuning out the noise: limbic-auditory interactions in tinnitus. Neuron 66:819-826

32. Roberts LE, Eggermont JJ, Caspary DM, Shore SE, Melcher JR, Kaltenbach JA (2010) Ringing ears: the neuroscience of tinnitus. J Neurosci 30:14972-14979

33. Rossi S, De Capua A, Ulivelli M, Bartalini S, Falzarano V, Filippone G, Passero S (2007) Effects of repetitive transcranial magnetic stimulation on chronic tinnitus: a randomised, crossover, double blind, placebo controlled study. J Neurol Neurosurg Psychiatry 78:857-863

34. Schlee W, Hartmann T, Langguth B, Weisz N (2009) Abnormal resting-state cortical coupling in chronic tinnitus. BMC Neurosci 10:11

35. Vandermeeren Y, Jamart J, Ossemann M (2010) Effect of tDCS with an extracephalic reference electrode on cardio-respiratory and autonomic functions. BMC Neurosci 11:38

36. Vanneste S, Plazier M, der Loo E, de Heyning PV, Congedo M, De Ridder D (2010) The neural correlates of tinnitus-related distress. Neuroimage 52:470-480

37. Vanneste S, Plazier M, Ost J, van der Loo E, Van de Heyning P, De Ridder D (2010) Bilateral dorsolateral prefrontal cortex modulation for tinnitus by transcranial direct current stimulation: a preliminary clinical study. Exp Brain Res 202:779-785

38. Vanneste S, Plazier M, van der Loo E, Ost J, Van de Heyning P, De Ridder D (2010) Burst transcranial magnetic stimulation: 
which tinnitus characteristics influence the amount of transient tinnitus suppression? Eur J Neurol 17:1141-1147

39. Wienbruch C, Paul I, Weisz N, Elbert T, Roberts LE (2006)

Frequency organization of the $40-\mathrm{Hz}$ auditory steady-state response in normal hearing and in tinnitus. Neuroimage 33:180-194

40. Ziemann U, Siebner HR (2008) Modifying motor learning through gating and homeostatic metaplasticity. Brain Stimul 1:60-66 